\title{
Exploring cellular memory molecules marking competent and active transcriptions
}

\author{
Li Xin ${ }^{\dagger}$, Guo-Ling Zhou ${ }^{\dagger}$, Wei Song, Xue-Song Wu, Gong-Hong Wei, De- \\ Long Hao, Xiang Lv, De-Pei Liu* and Chih-Chuan Liang
}

Address: From National Laboratory of Medical Molecular Biology, Institute of Basic Medical Sciences, Chinese Academy of Medical Sciences and Peking Union Medical College, Beijing, 100005, PR China

Email: Li Xin - xlgene@263.net; Guo-Ling Zhou - guolingzhou@gmail.com; Wei Song - songwei528@sohu.com; XueSongWu - wuxuesong@pumc.edu.cn; Gong-Hong Wei - liudp@pumc.edu.cn; De-Long Hao - liudp@pumc.edu.cn; Xiang Lv - liudp@pumc.edu.cn; De-Pei Liu* - liudp@pumc.edu.cn; Chih-Chuan Liang - liudp@pumc.edu.cn

* Corresponding author †Equal contributors

Published: 10 May 2007

BMC Molecular Biology 2007, 8:31 doi:|0.||86/|47|-2199-8-3|
Received: 7 September 2006

Accepted: 10 May 2007

This article is available from: http://www.biomedcentral.com/|47|-2/99/8/3।

(c) 2007 Xin et al; licensee BioMed Central Ltd.

This is an Open Access article distributed under the terms of the Creative Commons Attribution License (http://creativecommons.org/licenses/by/2.0), which permits unrestricted use, distribution, and reproduction in any medium, provided the original work is properly cited.

\begin{abstract}
Background: Development in higher eukaryotes involves programmed gene expression. Cell type-specific gene expression is established during this process and is inherited in succeeding cell cycles. Higher eukaryotes have evolved elegant mechanisms by which committed gene-expression states are transmitted through numerous cell divisions. Previous studies have shown that both DNase I-sensitive sites and the basal transcription factor TFIID remain on silenced mitotic chromosomes, suggesting that certain trans-factors might act as bookmarks, maintaining the information and transmitting it to the next generation.

Results: We used the mouse globin gene clusters as a model system to examine the retention of active information on M-phase chromosomes and its contribution to the persistence of transcriptional competence of these gene clusters in murine erythroleukemia cells. In cells arrested in mitosis, the erythroid-specific activator NF-E2p45 remained associated with its binding sites on the globin gene loci, while the other major erythroid factor, GATA-I, was removed from chromosome. Moreover, despite mitotic chromatin condensation, the distant regulatory regions and promoters of transcriptionally competent globin gene loci are marked by a preserved histone code consisting in active histone modifications such as $\mathrm{H} 3$ acetylation, $\mathrm{H} 3-\mathrm{K} 4$ dimethylation and K79 dimethylation. Further analysis showed that other active genes are also locally marked by the preserved active histone code throughout mitotic inactivation of transcription.
\end{abstract}

Conclusion: Our results imply that certain kinds of specific protein factors and active histone modifications function as cellular memory markers for both competent and active genes during mitosis, and serve as a reactivated core for the resumption of transcription when the cells exit mitosis. 


\section{Background}

Higher eukaryotes contain several hundred cell types, each with a distinctive gene expression pattern. During differentiation, a committed expression pattern is set up through the sequential turning on and/or off of different sets of genes and this pattern is propagated through numerous symmetric cell divisions. Thus, multicellular organisms have evolved mechanisms for discriminating their gene expression states and maintaining these stages during cell differentiation. When eukaryotic cells enter mitosis, the chromatin becomes highly condensed, most transcription factors are displaced from the chromosomes and almost all transcription is abruptly turned off [1]. How do eukaryotic cells remember their previous transcriptional profiles during cell division, rather than having to re-establish the appropriate expression pattern from scratch at the onset of the next cell cycle? In most situations, all cells carry the same genetic material, the genomic DNA, throughout their lives, so chromatinbased mechanisms including interactions among DNA, histones and non-histone protein factors and modifications of DNA and chromatin proteins may account for the transmission of gene expression states through cell division.

Previous studies have shown that DNase I-sensitive sites mark active genes on silenced mitotic chromosomes [2,3]. This implies that certain factors withstand chromatin condensation and survive during mitosis to facilitate the rapid reactivation of genes in next cell cycle. A subsequent study has supported this 'bookmarking theory' [4]. The basal transcription factor TFIID is partially retained on gene promoters in mitotic chromosomes to serve as such 'bookmark', enabling active transcription to be resumed rapidly on exit from mitosis $[5,6]$. However, the promoter alone may not be enough for the required memory. Many distant regulatory elements have been shown to play important roles in maintaining proper tissue- and developmental stage-specific transcription $[7,8]$. Some of these elements are DNase I-sensitive before the gene is activated. For example, in multilineage progenitor cells, erythroid-specific DNase I-hypersensitive sites (HSs) have arisen in the distal regulatory elements of the mouse $\beta$ globin gene cluster [9]. An early study showed that these hypersensitive sites, once induced, can be propagated to daughter cells (over at least 20 generations) in the absence of the original inducer [10]. We therefore speculated that in addition to TFIID, certain tissue-specific transcription factors might serve as 'bookmarks' on distant regulatory regions, which also mediate the propagation of chromatin states during cell division. In MEL cells, transcription of globin genes is not activated before induction, yet these genes can retain their transcriptional competence for generations. In this study, we used the globin gene clusters as a model system to investigate whether specific transcrip- tion factors remain on the distal regulatory elements in $\mathrm{M}$ phase chromosomes in transcriptionally competent MEL cells. We found that the p45 subunit of the erythroid-specific activator NF-E2 is partially retained on its specific binding sites during mitosis, but another activator, GATA1 , is not. This suggests that NF-E2 can function as a 'bookmark' to maintain the active competence of the local chromatin domain during cell division.

Recent advances in epigenetic studies have indicated that histone modification is also closely correlated with transcriptional regulation. Active histone modifications such as hyperacetylation and H3-K4 and H3-K79 methylation often mark transcriptionally active regions, whereas H3K9 methylation is always related to transcriptional repression. Combinations of histone modifications could constitute a set of histone codes that are recognized by specific non-histone proteins to mediate specific chromatin functions [11,12]. Using two continuously active hepatic genes as models, a recent study revealed that localized histone hyperacetylation, $\mathrm{H} 3-\mathrm{K} 4$ tri- or di-methylation and H3-K79 dimethylation at the promoter and the 5 ' portion of the coding regions remain stable during mitotic chromatin inactivation [13]. This suggests that epigenetic factors such as histone modifications should also be considered potential memory markers. Here we have investigated the active histone modification status on both the $\alpha$ - and the $\beta$-globin gene promoters and the distant hypersensitive sites on mitotic chromosomes in MEL cells. The results show that multiple active histone modifications are stably preserved on the mitotic chromosomes.

\section{Results \\ Erythroid-specific activator NF-E2, but not GATA-I, marking transcriptional competence of globin genes in MEL cell during mitosis}

In MEL cells, DNase I sensitivity and hypersensitivity have been established on globin gene loci [14], suggesting that they have obtained potential transcription activity. An early study also showed that hypersensitive sites, once been set up, can be propagated to daughter cells (over at least 20 generations) in the absence of the original inducer [10]. So we speculated that there might be some memory molecules, possibly involving protein factors or epigenetic marks, on regulatory sequences to help the propagation of transcription competence. There are some specific binding sites of erythroid-specific activators, such as GATA-1, NF-E2 and Fog-1, on globin gene clusters [15], whose occupancies largely contribute to the activation of globin genes. To explore the protein factors involved in the inheritance of transcriptional competence, we investigated the nuclear localization of GATA- 1 and the activating subunit p45 of NF-E2 in mitotic and interphase cell by in situ immunofluorescence. It was found that GATA-1 
mainly localized in interchromatin regions of cell nucleus and it can not be detected in nuclei of the mitotic cells by in situ immunofluorescence until parental nucleus divided into two progeny nuclei at telophase (Fig 1), indicating that GATA-1 was not retained on mitotic chromosome as a cellular memory mark during mitosis. In contrast, NF-E2p45, which is punctately organized in both cytoplasm and nucleus of the interphase cells, persists throughout mitosis (Fig 1), implying that it may be involved in the propagation of transcription competence of globin genes during cell division.

In order to confirm this possibility, we arrested murine erythroleukemia (MEL) cells into mitosis by treating the cells with nocodazole [3]. The mitotic index of cell population after treatment was confirmed to be $92-96 \%$ by flow cytometry analysis (Fig 2). The results also showed that a proportion of $5 \%$ mitotic cells, about $50 \%$ G0/G1phase and 45\% S-phase cells, respectively, is present in asynchronous MEL cell population (Fig 2). The two cell populations were analysed by ChIP to compare the occupancies of NF-E2p45 on distant hypersensitive sites of two mouse globin gene clusters (Fig 3A). The result showed that NF-E2p 45 could be specifically recruited to its binding sites HS26 and HS2 in interphase cells (Fig 3B). In mitotic cells it is still tightly associated with HS26 and HS2 (Fig 3B), indicating that NFE2p45 can be retained on mitotic chromosomes to maintain the hypersensitivity of the localized regulatory regions of two mouse globin gene

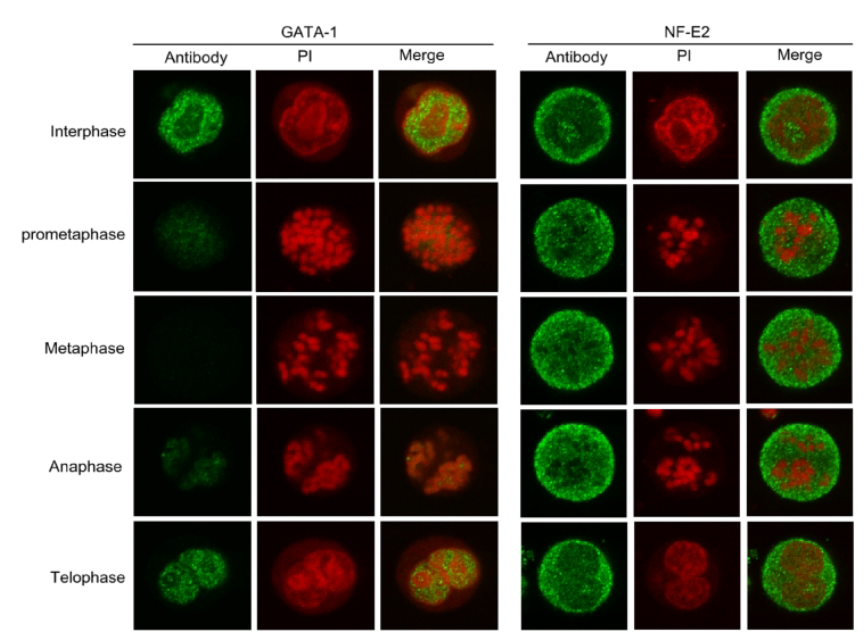

Figure I

Targeting of NF-E2p45 to chromatin compartment but displacing of GATA-I from mitotic chromosomes in mitotic cells. Cells were stained with antibodies against proteins indicated on the left followed by FITC-conjugated secondary antibodies (Left) and PI (Center). Right is the merge of both images. Examples of interphase cells and mitotic cells of different stages from MEL cells are shown. clusters. Its retention, moreover, marks the transcriptional competence of globin genes during MEL cell division.

\section{Epigenetic memory marking transcriptionally competent globin genes}

Besides protein factors, histone modification is recently identified as an efficient epigenetic factor involved in gene expression regulation through altering chromatin structure. Do the eukaryotic cells preserve some epigenetic marks on mitotic chromosomes to maintain its gene expression states? To verify the above-proposed possibility, we first observed the nuclear localization of four kinds of active histone modification including $\mathrm{H} 3$ acetylation, H4 acetylation, H3-K4 dimethylation and H3-K79 dimethylation in mitotic cells by in situ immunofluorescence. The results showed that all of the four modifications are retained on the mitotic chromosomes to some extent (Fig 4). We further detected the global changes of those modifications in both asynchronized and mitotic arrested MEL cell populations by western blotting. After correcting the variation in gel-loading and normalizing for the background, we found that there is a partial loss (about $20-30 \%$ ) of $\mathrm{H} 3$ acetylation, $\mathrm{H} 4$ acetylation and H3-K4 dimethylation in mitotic cell extraction compared to those in asynchronous cell extraction but no obvious loss of H3-K79 dimethylation (Fig 5). This indicates that only part of histone acetylation and H3-K4 dimethylation signals can be inherited to mitotic chromatin through DNA replication-coupled chromatin assembly process. What is the detailed distribution of these preserved modifications on the mitotic chromosome? We therefore analysed the histone modification status across the distant hypersensitive sites and the adjacent promoter regions of mouse $\alpha$ - and $\beta$-globin genes (Fig 3A) during mitosis by the comparative ChIP analysis of the above mentioned asynchronous and mitotic arrested MEL cell populations. The results showed that HS26, HS21 and HS8 of mouse $\alpha$ globin locus and HS3, HS2 and HS1 of mouse $\beta$-globin

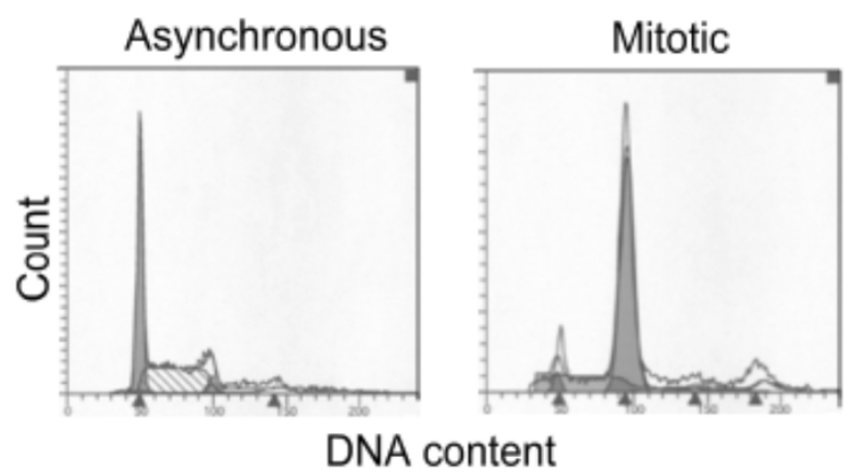

Figure 2

Flow cytometry analysis of asynchronous (A) and mitotic (M) MEL cells. 
A mouse $\alpha$-like globin gene cluster

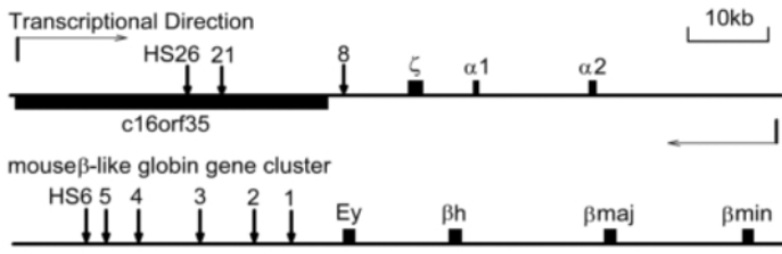

B

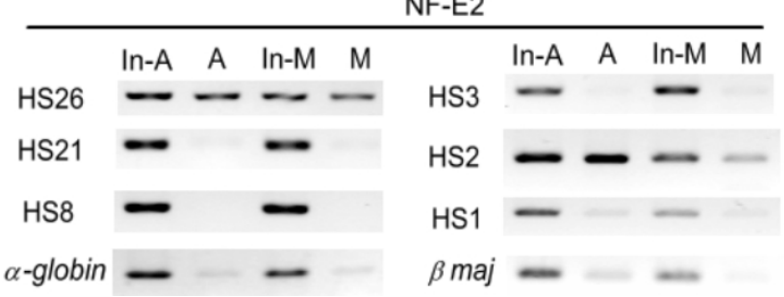

Figure 3

The occupancies of NF-E2p45 on mouse $\alpha$-like and $\beta$ like globin gene clusters in asynchronous $(A)$ and mitotic (M) MEL cells. A. The schematic presentation of mouse $\alpha$-like and $\beta$-like globin gene clusters. Globin genes are shown in dark rectangle. The DNase I hypersensitive sites-HS26, HS2I, HS8 of mouse $\alpha$-globin locus and HS3, HS2, HSI of mouse $\beta$-globin locus, which are analyzed in ChIP assay, are shown in arrow. $\mathrm{B}$. The relative occupancies of NF-E2p45 at HS sites of globin clusters in A and M cell populations were analyzed after normalizing the DNA amount differences in $A$ and $M$ cell populations through DNA input (In-A and In-M).

locus, as well as the promoters of $\alpha$-globin and $\beta m a j$ are acetylated at H3 and all of them except HS26 and HS3 are acetylated at $\mathrm{H} 4$ in asynchronous cells (Fig 6A, 6B). Higher H3-K4 dimethylation at HSs and $\alpha$-globin promoter was noticed when compared to that at $\beta$-globin promoter(Fig $6 \mathrm{C}$ ). HS 8 and $\alpha$-globin promoter are hypermethylated at H3-K79, but HS26 and HS21 show trace of signal. HS2 and HS1 of LCR are hypermethylated at H3K79, while HS3 and $\beta$ maj promoter are just slightly methylated at H3-K79 (Fig 6D). In mitotic cells, the levels of $\mathrm{H} 3$ and $\mathrm{H} 4$ acetylation, H3-K4 dimethylation dropped at many analyzed regions, while H3-K79 dimethylation at all the analysed regions remain stable compared to those in asynchronous cells. Moreover H3-K79 dimethylation level on mitotic chromosomes is comparable to that in asynchronous cells, which consist with their chromatin states (Fig 6). The results suggested that, despite of some losses, the established active histone code at distant regulatory regions and globin gene promoters before mitotic chromatin inactivation can be stably inherited to mitotic chromosomes to mark the transcriptional competence of mouse globin genes in MEL cells.

Epigenetic memory marking the active transcription states of the genes

Can these epigenetic marks note the different transcription states of thegenes during mitosis? We next performed
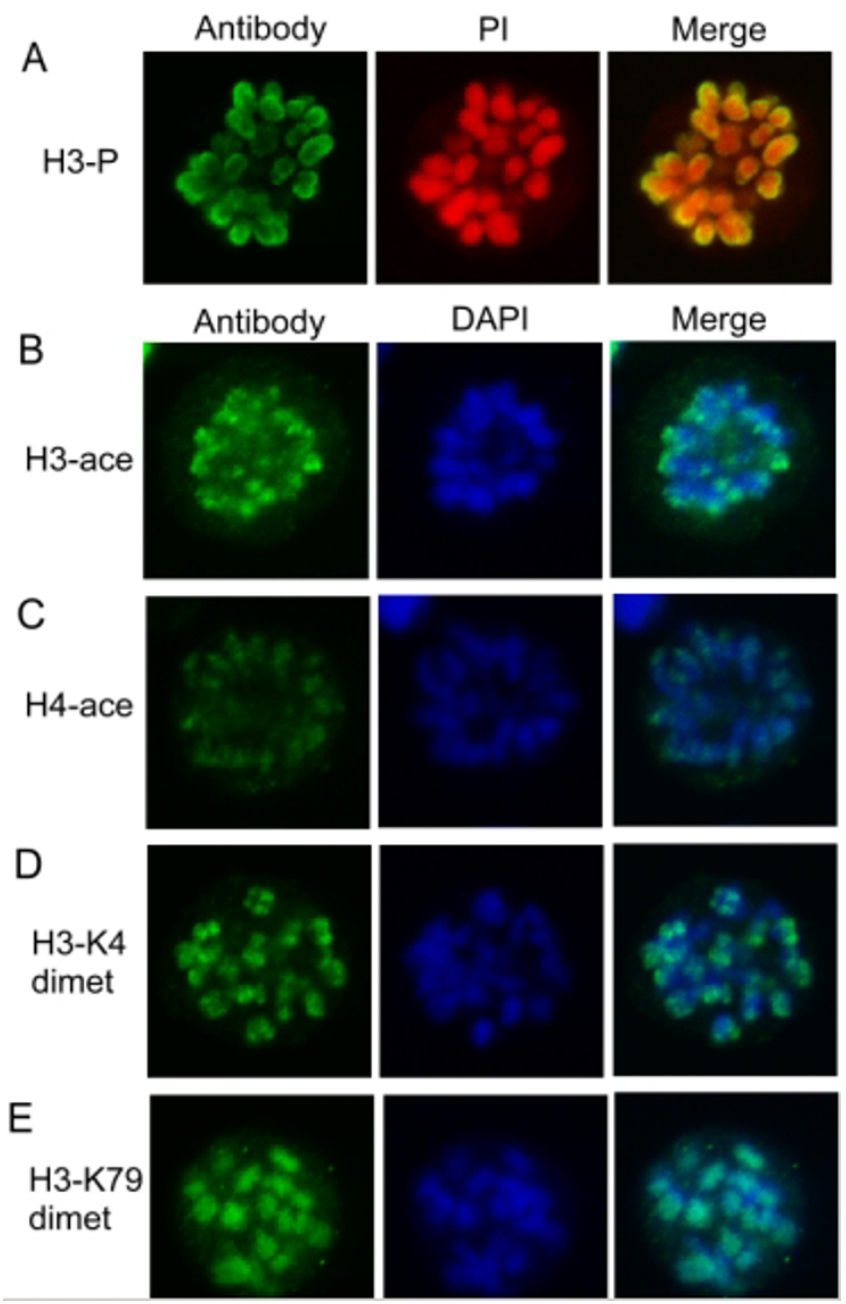

Figure 4

Flow cytometry analysis of asynchronous $(A)$ and mitotic (M) MEL cells. Cells were stained with antibodies against histone modifications indicated on the left followed by FITC-conjugated secondary antibodies (Left) and PI (red) or DAPI (blue) (Center). Right is the merge of both images. Mitosis marker-H3 phosphorylation $(\mathrm{A})$ and active histone modifications- $\mathrm{H} 3$ acetylation (B) and $\mathrm{H} 4$ acetylation (C), $\mathrm{H} 3$ $\mathrm{K} 4$ dimethylation (D) and K79 dimethylation (E) were observed on mitotic chromosomes by in situ immunofluorenscence.

the comparative chromatin immunoprecipitation (ChIP) analysis for the promoter regions of the genes with different transcription states. These genes include (1) $c$-myc, hsp70 and $\beta$-actin, which are universally transcribed by RNA polymerase II (RNAP II); (2) an actively RNAP IIItranscribed gene (7SK), and (4) nef-3 and albumin, which are tissue-specifically silenced in MEL cells. The results showed that promoter region of the actively transcribed genes like $c$ - $m y c$, $h s p 70, \beta$-actin and $7 S K$ are hyperacetylated, while inactive genes, nef-3 and albumin, are hypoacetylated at both $\mathrm{H} 3$ and $\mathrm{H} 4$ in asynchronous MEL cells (Fig 7A). In synchronized mitotic cells, transcription- 

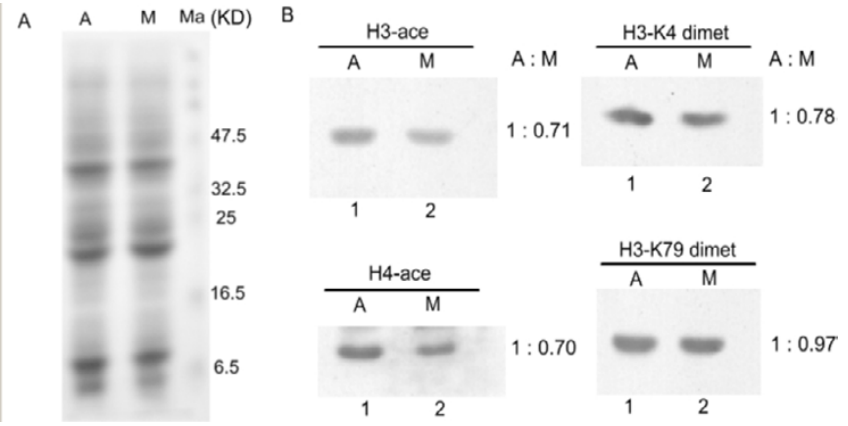

Figure 5

The comparative analysis of global histone modificartions in asynchronous (A) and mitotic (M) MEL cells through western blotting. A. Histones prepared from A and $M$ cell extracts were electrophoretically separated by SDS-polyacrylamide gel electrophoresis and stained with Coomassie Blue. B. The whole level of $\mathrm{H} 3$ and $\mathrm{H} 4$ acetylation, $\mathrm{H} 3-\mathrm{K} 4$ dimethylation in $\mathrm{M}$ cells is decreased compared to that in A cells, but H3-K79 dimethylation is stable.

ally active genes keep obviously higher levels of histone acetylation at both $\mathrm{H} 3$ and $\mathrm{H} 4$ than those of inactive genes,(Fig 7A). The fact that, during mitotic chromatin inactivation, the levels of remnant histone acetylation at the promoter regions of the analysed genes depend on their previous expression states strongly suggests that the localized histone acetylation at transcriptionally competent or active gene regions can be taken as the useful epigenetic memory marks to imprint the previous expression states during cell division.

To investigate whether active histone modifications-H3K4 dimethylation and K79 dimethylation are preserved at active regions during mitosis, we performed the similarly comparative ChIP analysis. The results showed that these two kinds of modification are also mainly confined to active gene promoters (Fig 7B). In asynchronous cells, the fully activated genes- $c-m \gamma c$ and $\beta$-actin are hyperdimethylated at H3-K4; incompletely activated gene hsp70 are moderately dimethylated at H3-K4. Although RNA polymerase III-transcribed gene $7 S K$ is active, similar to inactive genes as nef-3 and albumin, there is tiny enrichment of H3-K4 dimethylation on the gene. However, H3K79 dimethylation, despite the moderate level, is distributed on the promoters of all the analyzed active genes regardless of the RNA polymerase used for transcription. In mitotic cells, these two kinds of active histone modification, similar to histone acetylation, are also preserved on the previously active gene promoter regions and the preserved levels are corresponding with gene expression states. Nevertheless, there is some decrease for H3-K4 dimethylation but no obvious change for H3-K79 dimethylation (Fig 7B). These data indicate that the localized H3K4 dimethylation and K79 dimethylation at transcriptionally active regions can also serve as molecular memory
A

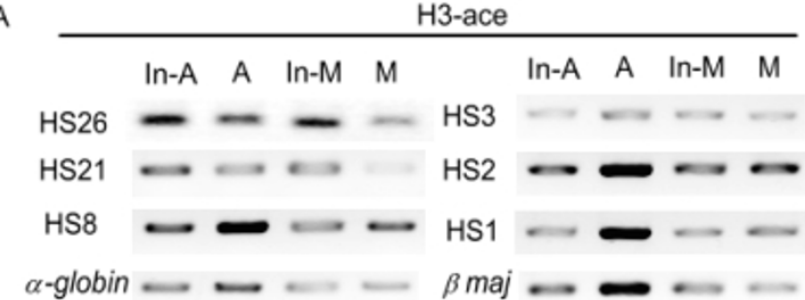

B

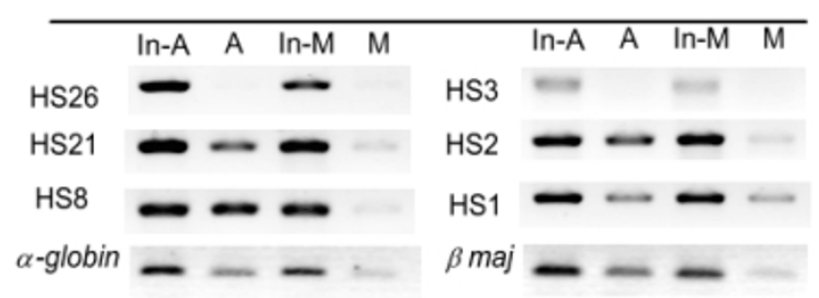

C

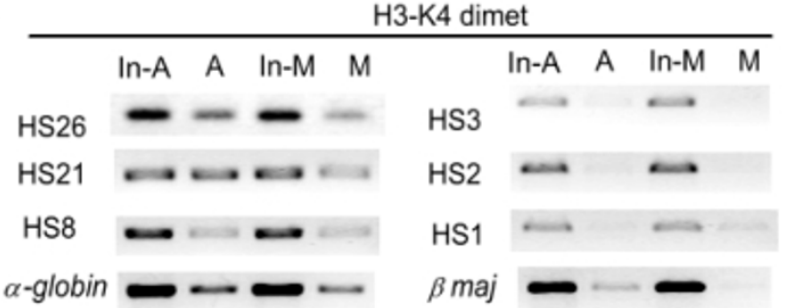

D

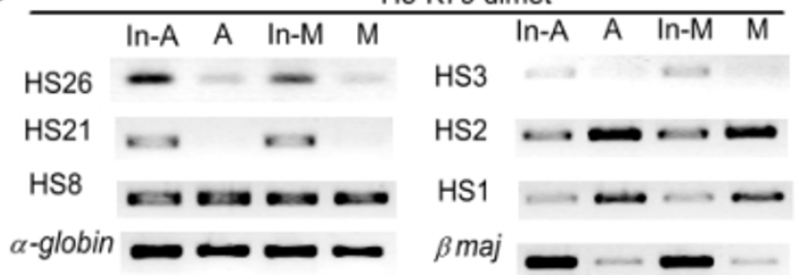

Figure 6

The distribution of active histone modifications on mouse $\alpha$-like and $\beta$-like globin gene clusters in asynchronized (A) and mitotic (M) MEL cells. A, B. The changes of $\mathrm{H} 3$ and $\mathrm{H} 4$ acetylation level on the distant regulatory elements of globin loci in $\mathrm{A}$ and $\mathrm{M}$ cell populations. $\mathrm{C}$, D. The changes of $\mathrm{H} 3-\mathrm{K} 4$ and $\mathrm{H} 3-\mathrm{K} 79$ dimethylataion on the distant regulatory elements of globin loci in $A$ and $M$ cell populations. The relative level of histone modifications at HS sites of globin clusters in $A$ and $M$ cell populations were analyzed after normalizing the DNA amount differences in $A$ and $M$ cell populations through DNA input (In-A and In-M).

marks along with histone acetylation. The above results also confirmed that the epigenetic marks composed of active histone modification combinations and localized at active gene regions can be transmitted to mitotic chromosomes for the maintenance of active gene expression states.

\section{Discussion}

Studies on the $\alpha$ - and $\beta$-globin genes suggest that certain mechanisms in higher eukaryotic cells fix the stochastic 

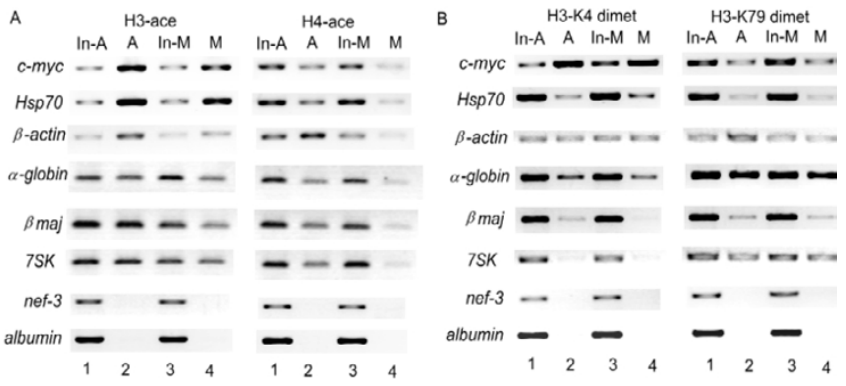

Figure 7

The distribution of active histone modifications on differently active gene promoters in asynchronized

(A) and mitotic (M) MEL cells. A. H3 and H4 acetylation. B. H3-K4 and H3-K79 dimethylation Comparative. ChIP analyses were performed with the equivalent DNA input amounts of chromatin from asynchronous and mitotic cells, then analyzed by PCR (In-A and In-M). PCR primer pairs flank the promoter regions of transcriptionally active, competent and inactive genes. The relative level of histone modifications at promoters of the individual genes in $A$ and $M$ cell populations were analyzed after normalizing the DNA amount differences in $A$ and $M$ cell populations through DNA input (In-A and In-M).

expression patterns during cellular differentiation. Although there is a tight balance in the expression of $\alpha$ and $\beta$-globin genes, these expression patterns are consistent with the predictions of the stochastic model $[26,27]$. Analysis has revealed an imbalance between $2 \alpha$ - and $2 \beta$ globin gene expression in both cytoplasm and nucleus in a significant proportion of erythroid cells. Further elegant experiments have demonstrated that these stochastic expression patterns are established prior to transcriptional activation. Importantly, both active and silenced expression patterns are clonally inherited [28]. The inherited stochastic patterns cannot be explained simply by random molecular encounters or fluctuations in the transitions between conformational states of a macromolecule, or by the amplification of feedback loops in a transcription factor network. Differentiated cells appear to have developed important mechanisms for remembering both transcriptionally active and silent states.

Previous studies have indicated that when cells enter mitosis, almost all transcription factors are peeled off the chromatin because the transcription complexes are disrupted [1]. However, it has been found that TFIID is retained at active gene promoters during mitotic chromatin inactivation [6]. During the process of transcription complex assembly, TFIID interacts first with the core promoter, so its retention on the mitotic chromosomes is an economical way of resuming transcription after mitosis. It has been observed that two enzyme classes, the histone acetyltransferases (HATs) and the histone deacetylases
(HDACs), are spatially reorganized and displaced from the condensing chromosomes as the cells progress through mitosis [16]. In another study [13] it was shown that histone acetylase (CBP, PCAF), chromatin remodeling complex component (Brg-1), SNF2H and FACT, along with RNA pol-II at the HNF-4, HNF-1 and albumin genes, are all dissociated from the chromatin during mitosis but re-recruited on mitotic exit [13], suggesting that they have roles in the reactivation of transcription.

By employing globin genes as a model system, we found in this study that the tissue-specific factor NF-E2p45, which is crucial for activating globin genes, is preserved on mitotic chromosomes but GATA- 1 is not. This suggests that during cell division, NF-E2p45 can serve as a molecular memory marker that maintains the locally hypersensitive state of the globin gene clusters. Previous studies have shown that GATA-1 is involved in regulating transcription by undergoing modifications such as acetylation, phosphorylation and sumoylation, without the recruitment of other partners [17-19]. Chromatin remodeling complexes and histone modification enzymes, which are displaced from mitotic chromosomes, depend on other protein factors to reunite them with the chromatin [13]. During globin gene activation, NF-E2 plays a key role in recruiting other protein factors such as $\mathrm{TAF}_{\mathrm{II}} 130$ and CBP, a coactivator of histone acetyltransferase activity [20]. TFIID retained on the mitotic chromosomes could also recruit other basal transcription factors when transcription is activated. Hence, we predict that higher eukaryotes have evolved mechanisms for preserving certain proteins on mitotic chromosomes, which may have common functional features for recruiting other protein factors to ensure that transcription is efficiently reactivated at the onset of each cell cycle. It will therefore be worth exploring other similar protein factors and their memory functions in order to elucidate cellular memory mechanisms.

The binding to DNA of many protein factors involved in the regulation of gene expression often depends on alteration of the local chromatin structure, mediated by posttranslational modifications of the terminal residues of histones. As a result, different histone modifications segregate the chromatin into different territories. Active chromatin regions are often hyperacetylated at histones $\mathrm{H} 3$ and $\mathrm{H} 4$ and hypermethylated at H3-K4 and H3-K79 [21]. However, active histone modifications are highly dynamic. When chromatin condenses during mitosis, therefore, do these modifications function as memory 'determinants' to mark different states of gene expression? In this study, we selected transcriptionally competent globin gene clusters and genes in different transcriptional states to investigate the potential role of four active histone modifications ( $\mathrm{H} 3$ and $\mathrm{H} 4$ acetylation, H3-K4 
dimethylation and K79 dimethylation) as cellular memory markers during mitosis, using comparative ChIP analysis of both asynchronous and mitotic MEL cell populations. In asynchronous cells, multiple combinations of the four histone modifications are located at the distant HSs of the globin gene clusters and the gene promoter regions, and they mark different chromatin states of the genes. For example, fully activated $c-m y c$ and $\beta$-actin are hyperacetylated at both $\mathrm{H} 3$ and $\mathrm{H} 4$ and hyperdimethylated at H3-K4; incompletely activated $h s p 70$ is hyperacetylated at both $\mathrm{H} 3$ and $\mathrm{H} 4$ and moderately dimethylated at $\mathrm{H} 3-\mathrm{K} 4$; potentially transcriptional $\alpha$ globin and $\beta m a j$ are moderately acetylated and dimethylated at $\mathrm{H} 3-\mathrm{K} 4$; the RNA polymerase III-transcribed gene $7 S K$ is hyperacetylated at both $\mathrm{H} 3$ and $\mathrm{H} 4$ but not dimethylated at H3-K4. Both transcriptionally active and competent genes, including RNA polymerase II- and IIItranscribed genes, are all moderately methylated at H3K79. However, inactive genes have less of these active modifications. In mitotic cells, some active modifications, especially $\mathrm{H} 3$ acetylation and $\mathrm{H} 3-\mathrm{K} 4$ dimethylation, are well preserved on the mitotic chromosomes. These preserved active histone modifications provide epigenetic markers by which gene expression states and local chromatin states can be inherited through mitosis. For the distant regulatory elements, which play important roles in switching on many tissue- or developmental stage-specific genes and maintaining their normal transcription during development and differentiation, localized active histone modifications, together with certain transcription factors, also provide important memory markers for maintaining local chromatin states during mitosis and hence facilitating and stabilizing differentiation and development. Moreover, the preserved epigenetic markers might contribute to create a unique chromatin conformation and provide a re-activating core for the resumption of transcription in the next cell cycle. They may also have roles in distinguishing transcriptionally active from repressed regions, even in the absence of trans-acting factors, at the onset of the next cell cycle.

Moreover, active modifications such as H3-K4 methylation and $\mathrm{H} 3$ acetylation are enriched in the $\mathrm{H} 3.3$ variant of Drosophila. In contrast, H3-K9 dimethylation, the marker for repressed genes, is enriched in H3 [22,23]. Another recent study showed that H3.3 is enriched in the promoters of active genes [24]. Using the mouse $\lambda 5$ VpreB1 locus as a model system, immuno-FISH analysis of mitotic cells revealed that H3.3 is strongly marked at the active $\lambda 5$ gene on metaphase chromosomes. Moreover, this region is hyperacetylated at $\mathrm{H} 3-\mathrm{K} 9$ and -K14 and di- and tri-methylated at H3-K4 [25]. These results strongly suggest that during mitosis, H3.3 might function as another epigenetic memory marker for maintaining transcriptionally active states along with active histone modification. Nevertheless, our speculation needs to be verified by testing more gene loci. It was also found that H3.3 incorporation into chromatin and its removal from chromatin depend on transcription [24]. Therefore, it will be worth exploring how H3.3 coordinates with multiple active histone modifications to mediate transcriptional memory.

\section{Conclusion}

We conclude that both specific transacting factors and active histone modifications could function as cellular memory markers for competent and active genes during mitosis, and could serve as a reactivated core for the resumption of transcription after the cells exit mitosis.

\section{Methods \\ Cell culture and mitotic arrest}

Murine erythroleukemia (MEL) cells were grown in Dulbecco's modified Eagle's medium (DMEM), supplemented with $10 \%$ fetal calf serum. The cells were arrested at the G2/M phase by treating with nocodazole (which synchronizes cells to metaphase [3] $\left(0.1 \mu \mathrm{g} \mathrm{ml}^{-1}\right.$, Sigma) for $16 \mathrm{~h}$. The mitotic index was determined by flow cytometry.

\section{Immunofluorescence}

The synchronized MEL cells were laid on the cover slips, and then fixed with 3.0\% paraformaldehyde at room temperature for $15 \mathrm{~min}$. Subsequently, cells were washed three times in PBS and then permeabilized in PBS containing $0.1 \%$ Triton X-100 for $5 \mathrm{~min}$. After that, cells were blocked with $3 \%$ BSA/PBS containing $2 \%$ goat serum followed by primary antibody incubation at $22^{\circ} \mathrm{C}$ for $2 \mathrm{~h}$ including the antibodies against GATA-1 (N6) and NFE2p45 (C-19) (Santa Cruz Biotechnology), histone H3 phosphorylation (06-570), H3 acetylation (06-599), H4 acetylation (06-866), H3-K4 dimethylation (07-030), H3-K79 dimethylation (07-366) (Upstate), which are diluted in blocking solution. The cover slips were washed three times in PBS, and then incubated in the presence of FITC-conjugated-anti-rabbit immunoglobin G (IgG) (F0382; Sigma) for a further $60 \mathrm{~min}$. Cover slips were washed again in PBS, incubated in $1 \mathrm{mg} / \mathrm{ml}$ DAPI or PI for 1 min and then mounted on slides with a $90 \%$ glycerolPBS (pH 7.5)-based medium containing $1 \mathrm{mg} / \mathrm{ml}$ paraphenylenediamine and sealed with nail polish. Immunostaining signal of cell preparations was recorded by Laser Screen Confocal Microscope (Bio-Rad, Randiance 2100) attached to a laser sharp software.

\section{Acid extraction of total histones and Western Blotting assay}

The asynchronous MEL and mitosis-arrested MEL cell populations were concentrated by centrifugation $(800 \times$ g) and washed twice with $1 \times$ PBS. The washed and con- 
centrated cells were resuspended in 5 volumes of ice-cold buffer (10 mM HEPES, pH 7.9, $1.5 \mathrm{mM} \mathrm{MgCl}_{2}, 10 \mathrm{mM}$ $\mathrm{KCl}, 0.5 \mathrm{mM}$ dithiothreitol, $1.5 \mathrm{mM}$ phenylmethylsulfonyl fluoride; Both dithiothreitol and phenylmethylsulfonyl fluoride were added just prior to the use of buffer). Concentrated sulfuric acid was added to the suspension to give a concentration of $0.4 \mathrm{~N}$. After incubation on ice for $30 \mathrm{~min}$, the suspension was centrifuged at $11,000 \times \mathrm{g}$ at $4^{\circ} \mathrm{C}$ for $15 \mathrm{~min}$. The resultant supernatant was decanted into another tube and mixed with $1 \mathrm{ml}$ of acetone. After overnight incubation at $-20^{\circ} \mathrm{C}$, the precipitated proteins were collected by centrifugation at $11,000 \times \mathrm{g}$ at $4^{\circ} \mathrm{C}$ for $15 \mathrm{~min}$. The pellet was washed twice with $1 \mathrm{ml}$ of acetone and air-dried. The dried pellet was then mixed with $2 \times$ SDS-polyacrylamide gel electrophoresis (SDS-PAGE) loading buffer. After incubation at $4{ }^{\circ} \mathrm{C}$ overnight, the supernatant was decanted into another tube and quantified for the western blotting assay.

For western blotting, the samples were boiled for $5 \mathrm{~min}$ and loaded onto a $15 \%$ SDS-polyacrylamide gel. The electrophoretically separated proteins were either stained with Coomassie Brilliant Blue or transferred to immnobilon-P membrane (Millipore). The membrane filters were blocked with PBST ( $1 \times$ PBS, $0.05 \%$ Tween 20$)$ containing 5\% nonfat milk powder (PBST-Milk) at room temperature for $1 \mathrm{~h}$ and then washed three times each with PBS-T for $15 \mathrm{~min}$. The primary antibody (anti-H3 acetylation: 1:10000, anti-H4 acetylation: 1:500, anti-H3-K4 dimethylation: 1:1000, H3-K79 dimethylation: 1:1000) diluted in PBST-Milk was applied at room temperature for $2 \mathrm{~h}$ or with agitation at $4{ }^{\circ} \mathrm{C}$ overnight. After three washes with PBS-T, the secondary antibody-goat anti-rabbit HRP conjugated $\operatorname{IgG}(12-348$, Upstate)(1:5000 dilution) in PBST-Milk was applied at room temperature with agitation for 1.5 hours. The filters were washed again and then developed using western blotting luminal reagent (SC2048, Santa Cruz Biotechnology) and exposed to film in darkroom for visualization.

\section{ChIP assay}

To crosslink chromatin, the cells were treated with $1 \%$ formaldehyde at room temperature for $10 \mathrm{~min}$. The reaction was stopped by the addition of glycine to a final concentration of $125 \mathrm{mM}$. The cells were washed with PBS and then the cell pellet was resuspended in SDS lysis buffer ( $1 \%$ SDS, 10 mM EDTA and $50 \mathrm{mM}$ Tris $\mathrm{pH} 8.1$ ) containing protease inhibitor cocktail (Roche). For one experiment, $1 \times 10^{6}$ cells in $200 \mu \mathrm{l}$ SDS lysis buffer were used for histone antibody and $1 \times 10^{7}$ cells in $200 \mu$ SDS lysis buffer for transcription factor antibody. The crosslinked cell lysate was sonicated on ice until crosslinked chromatin was sheared to DNA fragment length between 0.2 and $1 \mathrm{~kb}$. After centrifugation at $14000 \mathrm{r}$.p.m. for $15 \mathrm{~min}$, the $200 \mu \mathrm{l}$ sonicated cell supernatant was diluted 10 -fold by adding $1800 \mu \mathrm{l}$ ChIP Dilution Buffer (0.01\% SDS, 1.1\% Triton X-100, 1.2 mM EDTA, 16.7 mM Tris-HCl pH 8.1, $167 \mathrm{mM} \mathrm{NaCl}$, adding protease inhibitors as above). Meanwhile, another $20 \mu \mathrm{l}$ diluted cell supernatant was considered as input to quantitate the amount of DNA in different samples but it needed reverse the histone-DNA crosslinks by heating at $65^{\circ} \mathrm{C}$ for $4 \mathrm{~h}$. The $2 \mathrm{ml}$ diluted cell supernatant was precleared by incubation with $80 \mu \mathrm{l}$ of Salmon Sperm DNA/Protein A Agrose-50\% Slurry (16157 , Upstate) with agitation at $4{ }^{\circ} \mathrm{C}$ for $30 \mathrm{~min}$. The precleared chromatin was incubated with $4 \mu \mathrm{g}$ antibody with rotation at $4{ }^{\circ} \mathrm{C}$ overnight. All the used antibodies were the rabbit polyclonal antibodies against $\mathrm{H} 3$ acetylation (06-599), H4 acetylation (06-866), H3-K4 dimethylation (07-030), H3-K79 dimethylation (07-366) (Upstate), and against NF-E2 (C-19, Santa Cruz Biotechnology). For negative control, a no-antibody immunoprecipitation was performed by incubation of the supernatant fraction with $60 \mu \mathrm{l}$ of Salmon Sperm DNA/ Protein A Agrose-50\% Slurry (The results was not shown). Immunoprecipitation was recovered by incubation with $60 \mu \mathrm{l}$ of Salmon Sperm DNA/Protein A Agrose-50\% Slurry at $4{ }^{\circ} \mathrm{C}$ with rotation for $1 \mathrm{~h}$, followed by gentle centrifugation. The protein A agarose/antibody/histone complex was pelleted and washed with $1 \mathrm{ml}$ of each of the wash buffers for 5-10 min including low salt buffer, high salt buffer, and LiCl buffer (Upstate). Finally, it was washed twice using $1 \times$ TE. Then the pelleted protein A agarose/ antibody/histone complex was added $250 \mu$ freshly prepared elution buffer (1\%SDS, $0.1 \mathrm{M} \mathrm{NaHCO}_{3}$ ) and then incubated at room temperature with rotation for $15 \mathrm{~min}$ to elute the histone complex from antibody. The supernatant fraction (eluate) was transferred to another tube and elution was repeated. The $\sim 500 \mu$ l eluate was obtained to reverse crosslinks by incubation at $65^{\circ} \mathrm{C}$ for $6 \mathrm{~h}$ after adding $20 \mu \mathrm{l} 5 \mathrm{M} \mathrm{NaCl}$. The $10 \mu \mathrm{l}$ of $0.5 \mathrm{M}$ EDTA, $20 \mu \mathrm{l}$ of 1 $\mathrm{M}$ Tris- $\mathrm{HCl}, \mathrm{PH} 6.5$ and $2 \mu \mathrm{l}$ of $10 \mathrm{mg} / \mathrm{ml}$ Proteinase $\mathrm{K}$ (Roche) were added to the eluate and then it was incubated at $45^{\circ} \mathrm{C}$ for $4-5 \mathrm{~h}$. The DNA was recovered by phenol/chloroform extraction and ethanol precipitation (adding $20 \mu$ g glycogen). The pellet was washed with 70\% ethanol and dried in air. The dried pellet was diluted in 50 $\mu \mathrm{l} \mathrm{ddH}_{2} \mathrm{O}$ for PCR analysis. The primers used for ChIP analysis are listed in table 1 .

PCR products were resolved by agarose-gel electrophoresis and revealed by staining with ethidium bromide. For the comparative ChIP analysis, the linear range of amplification was determined for every sample by serial dilutions. An appropriate amount of DNA within the linear range was subsequently applied. All the comparative ChIP analyses were performed three repeats independently. 
Table I: The primers and primer sequences used in ChIP assay.

\begin{tabular}{|c|c|c|}
\hline $\begin{array}{l}\text { Primer } \\
\text { name }\end{array}$ & Primer sequences & Products \\
\hline c-myc & $\begin{array}{l}\text { 5'-CTGTCCTGGCTCGCAGATTGTAAG-3' } \\
\text { 5'-AGTGAGCGGACGGTTGGAAGAG-3' }\end{array}$ & $231 \mathrm{bp}$ \\
\hline hsp70 & $\begin{array}{l}\text { 5'-GTCGCAGCCAATCAACATAAGGATG-3' } \\
\text { 5'-GAAAGAGGCAGGAAACAAGTGACGC-3' }\end{array}$ & 229 bp \\
\hline$\beta$-actin & $\begin{array}{l}\text { 5'-GGACCGGCAACGAAGGAGCT-3' } \\
\text { 5'-GTCCACAAGGGCGGAGGCTAT-3' }\end{array}$ & $360 \mathrm{bp}$ \\
\hline$\alpha$-globin & $\begin{array}{c}\text { 5'-TTTGGTTTGAGGGACTTGCTTC-3' } \\
\text { 5'-TCATTGGCTGGTTTGTGCTTG-3' }\end{array}$ & $210 \mathrm{bp}$ \\
\hline$\beta$ maj & $\begin{array}{c}\text { 5'-ACCGAAGCCTGATTCCGTAGAGC-3' } \\
\text { 5'-GATGTCTGTTTCTGGGGTTGTGAGTC-3' }\end{array}$ & $269 \mathrm{bp}$ \\
\hline 7SK & $\begin{array}{c}\text { 5'-ACAGTCGGCTACATAAGGTCACCAC-3' } \\
\text { 5'-AGCTTCGGGAGGGACGCACA-3' }\end{array}$ & $213 \mathrm{bp}$ \\
\hline nef-3 & $\begin{array}{c}\text { 5'-TGATAAAGAACAGCGGACCCTACG-3' } \\
\text { 5'-AGTTCCAGACCTCTTGTCACCCG-3' }\end{array}$ & $228 \mathrm{bp}$ \\
\hline albumin & $\begin{array}{l}\text { 5'-CAGATGGCAAACATACGCAAGG-3' } \\
\text { 5'-GCAGAGCCGGAGACGAAGAG-3' }\end{array}$ & $246 \mathrm{bp}$ \\
\hline HS26 & $\begin{array}{l}\text { 5'-CGAGCAGATAACTAAGCCAAGC-3' } \\
\text { 5'-TTAAGGTAACAGGCACAGGGTC-3' }\end{array}$ & $185 \mathrm{bp}$ \\
\hline HS2I & $\begin{array}{c}\text { 5'-TGCCAGAATAACATCCGAGAACC-3' } \\
\text { 5'-GAGAGTCACTGATAGGAATGCCACC-3' }\end{array}$ & $17 \mid \mathrm{bp}$ \\
\hline HS8 & $\begin{array}{c}\text { 5'-CCTTATCTGTCCCGTCACTTGCTC-3' } \\
\text { 5'-ATTAATCTCGTCCATTCAGTTTCACC-3' }\end{array}$ & $250 \mathrm{bp}$ \\
\hline HS3 & $\begin{array}{l}\text { 5'-TGTTTCCCTGATGAGGATTCAATGG-3' } \\
\text { 5'-CCCACACATGGTCATCTATCTGAGC-3' }\end{array}$ & $274 \mathrm{bp}$ \\
\hline HS2 & $\begin{array}{l}\text { 5'-TTCCTACACATTAACGAGCCTCTGC-3' } \\
\text { 5'-AACATCTGGCCACACACCCTAAGC-3' }\end{array}$ & $279 \mathrm{bp}$ \\
\hline $\mathrm{HSI}$ & $\begin{array}{l}\text { 5'-GCCTTACATCCCTGGACAGATAAAC-3' } \\
\text { 5'-GTGACATTGTGAGAAACTGAGACCC-3' }\end{array}$ & 342 bp \\
\hline
\end{tabular}

\section{Authors' contributions}

LX conceived of the study, carried out experimental design, and participated in and molecular genetic study and drafted the manuscript. GLZ participated in the experimental design as well as carried out molecular genetic study and drafted the manuscript. XSW participated in cell synchronization and ChIP analysis, help to draft the manuscript. GHW participated in immunofluoresence study. WS participated in immunofluoresence study. DLH carried out cell culture. DPL conceived of the study, participated in coordination and helped to draft the manuscript. CCL coordinated and helped to draft the manuscript.

\section{Acknowledgements}

This work was supported by the grant from the Natural Science Foundation of China (Grant Number: 30393 II 0 and 3042 1003).

\section{References}

I. Gottesfeld JM, Forbes DJ: Mitotic repression of the transcriptional machinery. Trends Biochem Sci 1997, 22(6): 197-202.

2. Kerem BS, Goitein R, Diamond G, Cedar H, Marcus M: Mapping of DNAase I sensitive regions on mitotic chromosomes. Cell 1984, 38(2):493-499.

3. Michelotti EF, Sanford S, Levens D: Marking of active genes on mitotic chromosomes. Nature 1997, 388(6645):895-899.

4. John S, Workman JL: Bookmarking genes for activation in condensed mitotic chromosomes. Bioessays 1998, 20(4):275-279.

5. Chen D, Hinkley CS, Henry RW, Huang S: TBP dynamics in living human cells: constitutive association of TBP with mitotic chromosomes. Mol Biol Cell 2002, I3(I):276-284.

6. Christova R, Oelgeschlager T: Association of human TFIID-promoter complexes with silenced mitotic chromatin in vivo. Nat Cell Biol 2002, 4(I):79-82.
7. Nobrega MA, Ovcharenko I, Afzal V, Rubin EM: Scanning human gene deserts for long-range enhancers. Science 2003, 302(5644):4I3.

8. Spitz F, Gonzalez F, Duboule D: A global control region defines a chromosomal regulatory landscape containing the HoxD cluster. Cell 2003, I I3(3):405-4I7.

9. Palstra RJ, Tolhuis B, Splinter E, Nijmeijer R, Grosveld F, de Laat W The beta-globin nuclear compartment in development and erythroid differentiation. Nat Genet 2003, 35(2): 190-194.

10. Groudine M, Weintraub H: Propagation of globin DNAase Ihypersensitive sites in absence of factors required for induction: a possible mechanism for determination. Cell 1982, 30(1): $131-139$.

II. Jenuwein T, Allis CD: Translating the histone code. Science 200I, 293(5532): 1074-1080.

12. Turner BM: Cellular memory and the histone code. Cell 2002, I I I(3):285-29I.

13. Kouskouti A, Talianidis I: Histone modifications defining active genes persist after transcriptional and mitotic inactivation. Embo J 2005, 24(2):347-357.

14. Sawado T, Igarashi K, Groudine M: Activation of beta-major globin gene transcription is associated with recruitment of NF-E2 to the beta-globin LCR and gene promoter. Proc Natl Acad Sci U S A 200I, 98(18): I0226-I0231.

15. Anguita E, Hughes J, Heyworth C, Blobel GA, Wood WG, Higgs DR: Globin gene activation during haemopoiesis is driven by protein complexes nucleated by GATA-I and GATA-2. Embo J 2004, 23(I 4):284I-2852

16. Kruhlak MJ, Hendzel MJ, Fischle W, Bertos NR, Hameed S, Yang XJ, Verdin E, Bazett-Jones DP: Regulation of global acetylation in mitosis through loss of histone acetyltransferases and deacetylases from chromatin. I Biol Chem 200I, 276(4I):38307-38319.

17. Boyes J, Byfield P, Nakatani Y, Ogryzko V: Regulation of activity of the transcription factor GATA-I by acetylation. Nature 1998, 396(67II):594-598.

18. Collavin L, Gostissa M, Avolio F, Secco P, Ronchi A, Santoro C, Del Sal G: Modification of the erythroid transcription factor GATA-I by SUMO-I. Proc Natl Acad Sci U S A 2004, I 0 I (24):8870-8875.

19. Partington GA, Patient RK: Phosphorylation of GATA-I increases its DNA-binding affinity and is correlated with induction of human $\mathrm{K562}$ erythroleukaemia cells. Nucleic Acids Res 1999, 27(4): I |68-I I75.

20. Francastel C, Magis W, Groudine M: Nuclear relocation of a transactivator subunit precedes target gene activation. Proc Natl Acad Sci U S A 200I, 98(2I):12120-12125.

21. Schubeler D, MacAlpine DM, Scalzo D, Wirbelauer C, Kooperberg C van Leeuwen F, Gottschling DE, O'Neill LP, Turner BM, Delrow J, Bell SP, Groudine M: The histone modification pattern of active genes revealed through genome-wide chromatin analysis of a higher eukaryote. Genes Dev 2004, I8(II): | 263-127I.

22. Ahmad K, Henikoff S: The histone variant H3.3 marks active chromatin by replication-independent nucleosome assembly. Mol Cell 2002, 9(6): I I9I-I200.

23. McKittrick E, Gafken PR, Ahmad K, Henikoff S: Histone $\mathbf{H 3 . 3}$ is enriched in covalent modifications associated with active chromatin. Proc Natl Acad Sci U S A 2004, I 0 I (6): I 525-1530.

24. Schwartz BE, Ahmad K: Transcriptional activation triggers deposition and removal of the histone variant H3.3. Genes Dev 2005, I 9(7):804-814.

25. Chow CM, Georgiou A, Szutorisz H, Maia e Silva A, Pombo A, Barahona I, Dargelos E, Canzonetta C, Dillon N: Variant histone H3.3 marks promoters of transcriptionally active genes during mammalian cell division. EMBO Rep 2005, 6(4):354-360.

26. Trimborn T, Gribnau J, Grosveld F, Fraser P: Mechanisms of developmental control of transcription in the murine alpha- and beta-globin loci. Genes Dev 1999, 1 3: I I 2-124.

27. Wijgerde M, Grosveld F, Fraser P: Transcription complex stability and chromatin dynamics in vivo. Nature 1995, 377:209-213.

28. de Krom M, van de Corput M, von Lindern M, Grosveld F, Strouboulis J: Stochastic patterns in globin gene expression are established prior to transcriptional activation and are clonally inherited. Mol Cell 2002, 9:1319-1326. 